Editorial

\title{
Acknowledgement to Reviewers of Nutrients in 2014
}

Nutrients Editorial Office, MDPI AG, Klybeckstrasse 64, CH-4057 Basel, Switzerland

Published: 7 January 2015

The editors of Nutrients would like to express their sincere gratitude to the following reviewers for assessing manuscripts in 2014:

Abdelmalek, Manal F.

Abe, Sumiko

Abenavoli, Ludovico

Abner, Erin

Adelman, Daniel C.

Adjé, Félix

Adlard, Paul

Agho, Kingsley E

Aguilera-Tejero, Escolástico

Ahmed, Serge

Aisbett, Brad

Alam, Samina

Alda, Martin

Al-Dujaili, Emad

Alex, Sheril

Alexander, Szalai,

Alissa, Eman M.

Allen, Jason D.

Allott, Emma

Allsopp, Philip

Althuis, Michelle

Alwan, Na

Alway, Stephen E.

Amin, Rajesh

Andersen, Jens Rikardt

Andersen, Vibeke
Anderson, Alex Kojo

Anderson, John

Andersson, Helena

Andrade, Juan

Andrade, Paula Andrade

Andreoli, Angela

Angeloni, Cristina

Angulo-Barroso, R.M.

Annabi, Borhane

Annunziata, Azzurra

Anstey, Nick

Antonio, Joey

Aoi, Wataru

Apalset, Ellen M.

Apone, Fabio

Appleton, Katherine

Armanini, Decio

Armenta, Roberto

Armitage, Andrew E.

Arora, Pankaj

Arroo, Randolph

Aruoma, Okezie

Asard, Han

Aselage, Melissa B

Ashour, Mohamed

Atlantis, Evan
Augustin, Livia

Aukema, Harold M.

Aura, Anna-Marja

Auricchio, Salvatore

Austin, Gregory

Averna, M.R.

Azcona, Cristina

Baba, E.

Babatunde, Oyinlola

Bailey, Stephen

Bailo, Bibiana Garcia

Baker, Julien

Baker, Robert

Bakovic, Marica

Baldwin, Christine

Banu, Jameela

Baragetti, andrea

Barbagallo, Mario

Barbarossa, Iole Tomassini

Barclay, Alan W

Barker, Tyler

Barnett, Matthew P.G.

Barocelli, Elisabetta

Baron, Kelly Glazer

Barreira, João

Barton, Elisabeth R. 
Barvencik, Florian

Battino, Maurizio

Baumgartner, Jeannine

Beatty, Stephen

Beaven, C. Martyn

Beitz, Donald C.

Bellisle, France

Belski, Regina

BEMBEN, Michael G.

Bentley, David

Berendschot, Tos

Berge, Kjetil

Berger, Nathan A.

Berglund, Staffan

Berning, Jacqueline R.

Bertoia, Monica L.

Betts, Jonathan W.

Bhatia, Jatinder

Bielohuby, Maximilian

Birch, Edward

Bizzaro, Nicola

Bjorklund, Anneli

Bjornstad, Petter

Blanton, Cynthia

Blindauer, Claudia A.

Blusztajn, Jan

Boaz, Mona

Bogdanski, Pawel

Bogl, L.H.

Boisson-Vidal, Catherine

Booth, Alison

Borg, Søren

Borghi, Claudio

Börnhorst, Claudia

Bortolus, Renata

Bosy-Westphal, Anja

Boutsikou, Theodora

Bozzetti, Federico

Bozzetto, L.

Brabham, Brian

Bradbury, Kathryn E.

Braidy, Nady
Bramanti, Emilia

Brandhagen, Martin

Brand-Miller, Jennie C.

Brantsaeter, Anne

Braun, Barry

Bravi, Francesca

Breidenassel, Christina

Breit, Samuel N.

Bright, John J.

Brinkman, Maree T.

Brodribb, Wendy

Brophy, James M.

Brotherton, Carol S.

Brough, Louise

Brown, Andrew W

Brown, Jonathan

Brown, Lindsay

Brown, Rachel

Bryan, Nathan S.

Bryant, Eleanor J.

Bryszewska, Maria

Buchowski, Maciej S.

Buckland, Genieve

Buechler, Christa

Burdge, Graham

Burgess, John R.

Burmeister, Jacob

Burns, David

Buscemi, Silvio

Byrn, Mary

Cai, Yan

Calabrò, S.

Calder, Philip C.

Callaghan-Koru, Jennifer A.

Calvo, Mona

Campa, Adriana

Campbell, Norm R.C.

Campion, Bruno

Canas, Atilio

Candow, Darren

Canto, Carlos

Capewell, Simon
Carlberg, Carsten

Carless, Melanie A.

Carmel, Ralph

Caroli, Margherita

Carr, Anitra C.

Casanovas, Carmen

Cass, Wayne A.

Castaño, Angélica

Castaño, Paula M.

Castelao, Alberto Martinez

Castleman, Tony

Cederbaum, Arthur

Cermak, R.

Cervellati, Carlo

Cetinkaya, Ali

Chachay, Veronique S.

Chalmers, Kerry

Chan, Dick

Chan, K.K.L.

Chan, Maria

Chan, Queenie

Chan, Ruth

Chan, Yong Shin

Chand, Sourabh

Chang, Matt

Chaumontet, Catherine

Chelland Campbell, Sara

Chen, Chung-Yen

Chen, Chung-Yi

Chen, Ming-Hsuan

Chen, Ruei-ming

Chen, Tong

Chen, Yun

Chen, Zhe-Sheng

Cheng, HL

Cherniack, E. Paul

Childs, Emma

Chilibeck, Phil

Chilton, Robert J.

Chitchumroonchokchai, Julie

Chiu, Ken C.

Chmurzynska, Agata 
Cho, Youn-Ok

Christaki, Efterpi

Christopher, Gary

Chu, Fong Fong

Chun, Ock

Chung, Jay H.

Ciaccio, Edward J.

Ciancarelli, Maria Giuliana

Tozzi

Ciccone, Marco Matteo

Cicero, Arrigo F.G.

Cieślik-Boczula, Katarzyna

Clarke, Gerard

Claudia, Vetrani

Clemens, Roger

Coan, P.M.

Coates, Alison M.

Cobiac, Linda

Coe, Christopher L.

Coëffier, Moïse

Cohen, Ronald

Colby, Sarah

Collier, D.N.

Collins, Clare

Collins, James F.

Conlon, Beth A.

Conlon, Cath

Conrad, Kirk

Considine, Robert V.

Conterno, Lorenza

Conti, Ario

Cook, Brian

Cook, Nancy R.

Cooke, Matt

Corazza, Gino Roberto

Corbin, Karen

Cordero, Paul

Cordero-Macintyre, Zaida R.

Corley, M.M.

Cornish, Stephen

Costa-Rodrigues, João

Cotter, Joshua
Coudray, Charles

Cousins, Robert

Cozzolino, Mario

Crei, Franzcog

Crider, Krista S.

Cupisti, Adamasco

Cureton, Pamela A.

Curiel, J.A.

Curley, Robert

Curran, Christine

Custódio, Luísa

Da Graça Campos, Maria

da Silva, Robin P.

Dalen, Jeanne

Dalmas, Elise

Dalton, Caroline

D’Angelo, Stefania

Darmon, Nicole

Dasmahapatra, Asok

Dauncey, M.

Davidsson, Lena

Davis, Caroline

Davison, Glen

De Gara, Laura

De Marchi, Sergio

De Oliveira Otto, Marcia C.

De Souza, Russell

De Vries, Jeanne H.

Dean, Jeffrey A.

Decker, Eric A.

Dedoussis, George V.

Deldicque, Louise

Delgado-Calle, Jesus

Dellavalle, Diane M.

Delvin, Edgard E.

Deminice, Rafael

Denham, Bryan E.

Derave, Wim

Desai, $\mathrm{M}$.

Deut, Nicolaas E.P.

Devitt, Amy A.

Dew, Tristan
Dhillon, Varinderpal

Di Iorio, Biagio

Díaz Mejía, J. Javier

Diekman, Connie

Dik, Vincent

Dirkx, Ellen

Djilali-Saiah, Idriss

Dodd, J.

Doig, Gordon

Donadio, Carlo

Donovan, Sharon M.

Doyle, Todd

Draznin, Boris

DS, Wheeler

Duarte-Salles, Talita

Ducker, Kagan

Dugan, Mike

Duh, Pin-Der

Duncan, Robin

Dunford, Elizabeth K

Duntas, Leonidas

Duttaroy, Assim

Dyerberg, Jorn

Dyson, Pamela

Eaton, S.

Edirisinghe, Indika

Efird, Jimmy

Eichler, Klaus

Ekeroma, Alec

Eknoyan, Garabed

Elmståhl, Helena

El-Sohemy, Ahmed

Enck, Paul

Eneroth, Hanna

Engle-Stone, Reina

Erceg, David

Erinosho, Temitope O.

Escanero, Jesus Fernando

Esposito, C.

Evans, David C.

Eyles, Darryl

Eyles, Darryl W. 
Failla, Mark

Faith, Jeremiah

Fardet, Anthony

Fassett, Robert G

Faux, Noel

Fazeli, Pouneh

Feng, Lei

Ferguson, Lynnette

Ferguson-Stegall, Lisa

Fernandez, Maria Luz

Ferrando, Arny

Ferrer, Javier Izquierdo

Ferrie, Suzie

Field, Catherine

Fielding, Barbara

Filaire, Edith

Finamore, Alberto

Findlay, David

Finlayson, Graham

Fiorenza, Maria Teresa

Fitzgerald, Amanda

Fitzgerald, Kevin

Fitzgerald, Richard J.

Foerster, Jana

Foo, Leng Huat

Foreyt, John

Forhead, Alison J.

Fort, Patrice

Foster, J.A.

Foster, Meika

Franci, David S.

Frazier, Elisabeth

Freedman, Marjorie

Freisling, Heinz

Fresco, P.

Friedman, Jacob E.

Friesen, Carol

Fu, Tzu-Fun

Fu, Zhengwei

Fuglestad, Anita J.

Fukami, Kei

Fukazawa, Masanori
Fukuda, Michio

Fukuda, Mitsuru

Funato, Hiromasa

Funtikova, Anna

Furtado, Kelly

Gadella, Bart M.

Gagnon, Dominique D.

Gahete, Manuel D.

Galderisi, Umberto

Gallaher, Daniel D

Gallo, Valentina

Garcia, Diego

Garcia-Larsen, Vanessa

Garden, Frances

Gardiner, Paula M.

Gareau, Mélanie G.

Garinis, George

Gatterer, Hannes

Gearhardt, Ashley N.

Geelen, Anouk

Gérard, Philippe

Ghadimi, Darab

Ghosh, Rita

Giannessi, Daniela

Gibson, Deanna

Gibson, Peter R

Gill, Chris Ir

Giltay, Erik

Giovannini, Claudio

Giudetti, Anna

Glickman, Ellen

Glocker, Erik-Oliver

Gogorcena, Yolanda

Going, Scott B.

Gombart, Adrian

Gómez-Ambrosi, Javier

Gómez-Gómez, Lourdes

Gong, Jian

González-Navarro, Herminia

Goodwin, Huw

Gormaz, Juan G.

Graham, Dan J.
Grant, Ross S.

Grant, William

Grassi, Davide

Greatwood, H.C.

Grebenstein, Patricia E.

Greco, Luigi

Green, Tim

Grieger, Jessica

Griffin, Ian

Grimshaw, Kate E.C.

Gross, Micah

Grundmann, Oliver

Guasch, Marta

Gueimonde, Miguel

Guerzoni, Maria Elisabetta

Guillem, Karine

Gupta, Sanjay

Haase, Hajo

Hafeez, Bilal Bin

Hafekost, Katherine

Hagobian, Todd

Halade, Ganesh

Hamden, Khaled

Hammond, Billy

Han, Dongmei

Han, Sung Nim

Hanhineva, Kati

Hanna, Katherine

Haque, Azizul

Harding, Scott

Harland, Janice

Haroutounian, Serkos A.

Harrington, Jennifer

Harris, Cristen

Harris, William

Harris, William S.

Harrison, Fiona

Hart, Prue

Hartshorn, Kevan

Hasegawa, Daisuke

Haskell, Crystal

Hasty, Dr. Alyssa H. 
Hattori, Satoshi

Haugen, Margaretha

Hauner, Hans

Hawley, John A.

Hay, William

He, Tao

Heaney, Robert

Heber, David

Hector, Debra

Hegazi, Refaat A.

Heine, Gunnar H.

Heinemann, Akos

Heinrich, Ulrike

Hellhammer, Juliane

Hemila, Harri

Hemsworth, Jaimie

Hennessy, Áine

Hennigar, Steve

Hinckson, Erica

Hipgrave, David B.

Hixson, Stefanie M.

Hobin, Erin

Hocquette, Jean-François

Hoffman, Sarah R.

Hoffmann, Kristina

Hokama, Tomiko

Holick, Michael F.

Hollis, Bruce

Holvik, Kristin

Hong, Eock Kee

Hong, Mee Young

Hook, Ingrid L.I.

Hooshmand, Shirin

Hopkins, Will

Houghton, C.A.

Houghton, Lisa A.

Houston, Mark C.

Hruby, Adela

Hsing, Chung-Hsi

Huang, Tsui-Chin

Huang, Yi-Chia

Hudson, André O.
Hughes, David

Hugo Teixeira, Vitor

Hulthén, Lena

Hummel, Sandra

Hung, Kuan-Yu

Hunter, J.O.

Hurst, Jeffrey

Husby, Steffen

Hutchins, Andrea

Hyde, Matthew

Ibiebele, Torukiri

Illner, A.

Imamura, Hiroyuki

Imperatori, Claudio

Iriti, Marcelo

Islam, M. Munirul

Itoh, Y.

Itsiopoulos, Catherine

Ivey, Kerry L.

Iwasaki, Masanori

Jaacks, Lindsay M.

Jackson, Phillipa

Jakubowicz, Daniela

Järvinen, Tero

Jean-Marc Tadié, Jean-Marc

Jeewon, Rajesh

Jeffery, Ian

Jeffry, Paul

Jiménez-Chillarón, Josep C.

Johner, Simone A.

Johnson, Ian

Johnson, Rachel

Johnston, Carol

Johnston, Craig

Jones, Andrew M.

Jones, Julie

Joosten, Etienne

Jorde, Rolf

Joven, Jorge

Joy, Edward J.M.

Ju, Sang-Yhun

Judd, Suzanne
Jun, Dae Won

Juturu, Vijaya

Kaciroti, Niko

Kahn, Richard

Kajiemail, Hiroshi

Kaklamani, Virginia

Kalliomäki, Marko

Kamada, Nobuhiko

Kamphuis, Patrick

Kane, Maureen A.

Kang, Sinyoung

Kano, Mitsuyoshi

Kanoski, Scott E.

Kaplan, Birsen

Kaplan, Bonnie J.

Karin, Amrein

Karlic, Heidrun

Karpinski, Christine

Karras, S.N.

Karunasinghe, Nishi

Kasperzyk, Julie L.

Kaster, Manuella

Kataoka, Yosky

Katayama, Shigeru

Kattelmann, Kendra

Katzberg, Hans Dieter

Kaunitz, Jonathan D.

Kavouras, Stavros

Kawakami, Shinpei

Kay, Colin

Kaye, Elizabeth Krall

Keast, Russell

Keim, N.L.

Kelley, Darshan

Kelley, Darshan S.

Kelley, Katherine

Kelley, Melissa D.

Kennedy, Deborah A.

Kenny, Anne

Kenyon, Nicholas J.

Kern, Benjamin

Key, Timothy J 
Khalafallah, Alhossain A.

Khanal, Vishnu

Khymenets, Olha

Kido, Teruhiko

Kim, Byeong Mo

Kim, Cristina

Kim, Hail

Kim, Jongkyung

Kim, Mina K.

Kim, Tack-Joong

Kim, Young-jae

Kim, Yun-bae

Kingsley, Michael

Kirkpatrick, Sharon I.

Kitatani, Kazuyuki

Kłęk, Stanisław

knaapila, Antti

Knackstedt, Lori A.

Knechtle, Beat

Kniefel, Wolfgang

Ko, Hyun-Jeong

Ko, Kam Ming

Koehler, Peter

Koh, Timothy

Kojima, Masayasu

Kojo, Shosuke

Kolovou, Genovefa

Kongkachuichai, Ratchanee

Koolen, Hector Henrique

Ferreira

Koot, Bart G.P.

Kopecky, Jan

Kordas, Katarzyna

Kotzka, Jorg

Kovacevic, Zaklina

Krämer, Klaus

Krebs, Nancy

Kreichauf, Susanne

Kreider, Richard

Kroeker, Karen I.

Kubis, Hans-Peter

Kuhnle, G.G.C.
Kumar, Matam Vijay

Kumar, Yogesh

Kundur, Avinash Reddy

Kuo, Shiu-Ming

Kurokawa, Masahiko

Kussmann, Martin

Kwak, Chung Shil

Kwon, Oran

Lacaille-Dubois, Marie-Aleth

Lacan, Dominique

LaFleur, Susanne

Laguna, Juan C.

Lam, Yan Y.

Lambert, Jennifer

Lanaspa, Miguel A.

Land, Mary-Anne

Lanigan, Julie

Lanou, Amy Joy

Lanthier, Nicolas

Laplante, Mathieu

Larson-Meyer, Enette

Lassek, William

Latunde-Dada, Gladys O

Laughlin, Maren R.

Laverty, Anthony A.

Lawton, Clare L.

Leclerc, Marion

Lee, Chia-Lun

Lee, Chung-Yung Jetty

Lee, Hyeon Yong

Lee, Ki Won

Lee, Kwang Ho

Lee, Myoungsook

Lee, Terence Kin-Wah

Leech, Rebecca M.

Leem, Kang-Hyun

Lei, Xin-Gen

Lemieux, Simone

Lennox, Alison

Lent, Michelle

Lentjes, Dr. Marleen

Lerner, Ulf
Leung, Ping-Chung

Levy, Yishai

Lewis, James D.

Li, Bin

Liem, Gie

Lillioja, Stephen

Lin, Ding-bo

Lin, Jin-Ding

Lin, Ping-Ting

Lionetti, Elena

Little, Jonathan P.

Liu, Biing-Hui

Liu, Peggy

Locatelli, Francesco

Lodemann, U.

Lodge, John

Lonardo, Amedeo

Longo, L.D.

López-Carballo, Gracia

Lophatananon, Artitaya

Loprinzi, Paul

Lord, Janet

Lorentz, Axel

Lorenz, Mario

Lorenzi, Rodrigo

Lorini, Dott.ssa C.

Louboutin, Jean-pierre

Lu, Chen

Lu, Jing

luca, Busetto

Lukowski, Angela F.

Lusis, Aldons J.

Lutsey, Pamela L.

Luyckx, Valerie A.

Ma, David W.L.

Ma, Jin Yeul

Macdiarmid, Jennie

Macdonald, Jamie Hugo

MacFarlane, Amanda J.

Mackenzie, Lincoln

Macpherson, Helen

Madar, Zecharia 
Madej, Dawid

Maes, Michael

Magann, Everett F.

Maggini, Silvia

Maggio, Albino

Magnusson, Roger

Maher, Timothy J.

Mair, Christiane

Maitland, Kathryn

Makarenko, Vladislav

Mallard, Simonette R.

Manco, Marco

Mangialasche, Francesca

Mann, Samuel J.

Mantovani, Giovanni

Marakis, Georgios

Marek, Ryan J.

Marian, Mary

Mariotti, François

Markhus, Maria Wik

Martel, Fátima

Martínez González, Miguel

Ángel

Martínez-Cuesta, M. Carmen

Martínez-Micaelo, Neus

Maruvada, Padma

Mas, Emilie

Maslova, Ekaterina

Masulli, Maria

Matsumoto, Ippei

Matsumoto, K.

Matsumura, Kenta

Matsuoka, Yutaka

Mattar, Melanie

Mattijssen, Frits

McArthur, Jennifer O.

McCarthy, David

McCarthy, John

McCarty, Catherine A.

McCarty, Mark

Mcclung, James

McConell, Glenn
McDaniel, John

Mcdougall, G.J.

McDougall, John

McGrath, John J.

McGrath, Kristine

McIntosh, Graeme

McKenna, Malachi J.

McManus, Alexandra

McQuestion, Maurene

Mecocci, Patrizia

Medlock, Amy

Mehta, Rajendra G.

Meijers, Joost C.M.

Mekhedov, Sergei

Meli, Rosaria

Memon, M.A.

Mencarelli, Fabio

Mennella, Julie

Meredith, M. Elizabeth

Messner, Donald J.

Meyer, Anne

Michels, Alexander J.

Miguel, M.G.

Miller, Joshua W.

Miller, Gary D.

Miller, Matt

Miller, Matthew

Miller, Tracie L.

Miller, Woodgate

Milte, Catherine

Minutolo, Roberto

Miraglia, Emanuele

Mischley, Laurie

Mishra, Suman

Mishur, Robert J.

Mo, Huanbiao

Moffat, Tina

monro, John.

Moody, A. John

Moon, Jordan

Moore, Amanda

Morales, Eva
Morales-González, José

Moran, Carthage P.

Morelli, Lorenzo

Moretti, Diego

Moriyama, Tatsuya

Morris, Howard

Morteza, Afsaneh

Mounier, Catherine

Mountjoy, Margo

Muhlhausler, Beverly

Mukai, Yuuka

Mukhar, Hasan

Müller, W.E.G.

Mulligan, Angela A

Mullin, James M.

Munoz, Colleen

Murakami, Haruka

Murff, Harvey J.

Mustacich, Debbie J.

Mutti, Elena

Mwatsama, Modi

Nagaoka, Satoshi

Nagineni, Chandrasekharam N.

Nair, Krishnapillai Madhavan

Nairz, Manfred

Nakahama, Kenichi

Nammi, Shrinivas

Naska, Androniki

Navarro, Carlos Gonzalez

Nelson, Michael

Netticadan, Thomas

Neu, Josef

Neumann, Charlotte

Newsholme, Philip

Neyraud, Eric

Nguyen, Douglas L.

Nicoletti, Claudio

Nieman, David C.

Nievar, M. Angela

Nijdam, Durk

Niki, Etsuo

Nikolaidis, Michalis G. 
Nino, Russo

Nissim, Ahuva

Nosova, Emily

Nwaru, Bright

Oba, Shino

Oberhelman, Sara

Obrenovich, Mark E.

O'Connell, Thomas

O’connor, Helen T.

Oda, Hiroaki

O’Dell, Sandra

Oelkrug, Christopher

Oesterreicher, Christoph

Ogata, Tadanori

Oglesby-Sherrouse, Amanda

Oinonen, Kirsten A.

Ojeda, N.B.

O'Keefe, Stephen J.D.

Olefsky, Jerrold M.

Olek, Rovert A.

Onakpoya, Igho J.

Onda, Ken

O'neil, Carol

Ormsbee, Michael

Ornish, Dean

Osher, Yamima

O'Sullivan, Aifric

Oterhals, Åge

Pacanowski, Carly

Pacetti, Deborah

Pachon, Helena

Pacilli, Maurizio

Page, Amanda J.

Paiva-Martins, Fátima

Pala, Valeria

Panahi, Shirin

Pantano, Kathleen Joan

Paoli, Antonio

Papadakis, Sophia

Papadimitriuou, Konstantinos

Papadopoulou, Eleni

Papetti, Adele
Park, Hwa-Jin

Park, Kyong

Park, Yongsoon

Parletta, Natalie

Parthasarathy, Sampath

Patten, Glen S.

Patterson, Bruce

Patterson, Michael

Payne, Martha

Peairs, Abigail D.

Peake, Jonathan

Pedram, Pardis

Peñagaricano, Francisco

Peng, Guang

Pepino, M. Yanina

Perks, C.M.

Peter Stehle, Peter

Péterfy, Miklós

Peterson, Catherine A.

Peterson, Julia

Peterson, Linda

Pezzarossa, Beatrice

Phillips, Stuart M.

Pinto, John T.

Pittman, Karin

Plösch, Torsten

Plourde, Mélanie

Poblaciones, Maria J.

Polidori, Maria Cristina

Pothos, Emmanuel N.

Pottala, James

Powers, Hilary J.

Powles, John W.

Pradelli, Lorenzo

Premkumar, Muralidhar

Pritchard, Janet

Privitera, Gregory

Qi, Lu

Qin, Bolin

$\mathrm{Qu}, \mathrm{Yan}$

Quatromoni, Paula

Raatz, Susan K.
Rachek, Lyudmila

Radmacher, Paula

Ragaee, S.M.

Raha, Sandeep

Raj, Sudha

Ranadheera, C. Senaka

Randall, Philip

Randall-Simpson, Janis

Rasmussen, Nicolas

Rasperini, Giulio

Rattan, Sureshi. S.

Rauch, Bernhard

Reed, Bruce

Rehm, Colin

Reid, Gregor

Reimer, Raylene A.

Reis, Joanna

Reis, Mariza G.

Remacha, A.F.

Rezamand, Pedram

Rigottier-Gois, Lionel

Rhodes, Jonathan

Ridoutt, Brad

Rijntjes, Eddy

Riley, Malcolm D.

Riverin, Bruno

Roa Romero, Laura M.

Roberts, Michael

Robertson, Graham

Roche, $\mathrm{M}$.

Rodriguez, Ana

Rodriguez-Rodriguez, Rosalia

Rogers, Lynette K.

Rombouts, Ine

Romero, Maria-Paz

Rondanelli, Mariangela

Root, Martin

Rosa, Angelo

Rose, Devin

Rosendale, Douglas Ian

Ross, A. Catherine

Ross, Alastair B. 
Rossi, Mauro

Rothers, Janet

Rousseau, Anne-Sophie

Rousseau, G.

Rozowski, Jaime

Rucklidge, Julia

Rudkowska, Iwona

Rurak, Dan

Rush, Elaine

Rust, Rosanne

Ruxto, Carrie

Ryan, Lisa

Rybalka, Emma

Ryu, Jae-Ha

Sachan, Dileep S.

Saheki, Takeyori

Sakuma, Kunihiro

Sale, Craig

Salmerón, Ivan

Salvini, Filippo

Samocha-Bonet, Dorit

Sanchez-Muniz, Francisco

Sandoval, Claudio

Sangild, Per T.

Santini, Antonello

Sapuntzakis, Maria

Sartore, Giovanni

Sasazuki, S.

Satarug, Soisungwan

Sathishkumar, K.

Sato, Kenji

Sato, Shin

Satta, Yoko

Savica, Vicenzo

Saw, Constance Lay Lay

Schiavone, Stefania

Schini-Kerth, Valérie

Schlienger, Jean-Louis

Schmidt-Heydt, Markus

Schoeler, Natasha E.

Schroeder, Helmut

Schubert, Matthew M.
Schultz, Clyde L.

Schulze, Kerry

Schupp, Michael

Schweitzer, Dietrich

Scoffield, Jessica

Scott, Dr. Hayley

Scragg, Robert

Sellke, Frank W.

Seluanov, Andrei

Senchina, David

Seo, Hyung Seok

Serio, Francesco

Serra, Carlo

Serra, Serra

Serralheiro, Maria Luísa M.

Serra-Majem, Lluís

Serrano, María

Serrat, Maria A.

Seth, Anju

Shahab-Ferdows, Setti

Shakur, Yaseer A

Sharoni, Yoav

Shen, Chwan-Li

Shertzer, Howard G.

Shi, Zumin

Shiloach, Joseph

Shimizu, Takahiko

Shiva, Sruti

Shoben, Abigail B.

Shrapnel, Bill

Siafarikas, Aris

Siddappa, Asha Jyothi

Silver, Heidi J.

Simeonov, V.

Simopoulos, Artemis

Siniscalco, Dario

Sioen, Isabelle

Sjögren, Per

Skinnert, Tina

Skipworth, Richard J.E.

Skov, Laurits

Smedslund, Geir
Smith, Adam

Smith, Caren E.

Smith, E.

Smith, Kylie J.

Smith, Lindsey

Sørensen, L.B.

Specker, Bonny

Spedding, Simon

Srzednicki, George

Stachowicz-Stencel, Teresa

Staege, Martin S.

Staiano, Anna-Maria

Stanhope, Kimber

Stefanidou, Maria E.

Stefanska, Barbara

Stefanutti, Claudia

Steinberg, Francene M.

Steinle, Nanette I.

Stephen, Mary C.

Sterkowicz, Stanislaw

Stevenson, D.E.

Stilli, Donatella

Stone, William

Strain, Sean

Strandvik, Birgitta

Strauss, William

Su, Hui-Min

Su, Xiao

Subramanian, Veedamali S.

Sugatani, Junko

Sugimoto, Ken

Sugiyama, Kemmyo

Suh, Miyoung

Sun, Guang

Sun, Hui Sun

Sung, Ping-Jyun

Szeto, Yim Tong

Takahisa, Kanekiyo

Takeda, Norifumi

Tako, Elad

Tamura, Tsunenobu

Tan, S.-Y. 
Tanaka, Hiroyuki

Tanofsky-Kraff, Marian

Tappia, Paramjit S.

Tappy, Luc

Tashiro, Hirotaka

Taylor, Lem

Teas, Jane

Temple, Jennifer L.

Tepper, Beverly J.

Terai, Shuji

Terrin, Gianluca

Tessitore, Antonio

Thelen, Paul

Thèvenod, Frank

Thoma, Christian

Thomas, David Travis

Thomas, Lynn K.

Thunders, Michelle

Tibolla, Gianpaolo

Tiidus, Peter M.

Timmerman, Kyle

Tlaslakova-Hogenova, Helena

Tobwala, Shakila

Tomofuji, Takaaki

Torheim, Liv Elin

Tosi, Paola

Tou, J.C.

Tran, Minh-Ha

Transler, Catherine

Travagli, Valter

Trayhurn, Paul

Tremblay, Angelo

Triantafillidis, John K.

Trosko, James E.

Tsai, Ying Chieh

Tsao, Po-Nien

Tscholl, Philippe

Tsiplakou, E.

Tsoli, Maria

Tsuji, Petra

Tulchinsky, Ted

Túnez, Isaac
Turon, X.

Tussing-Humphreys, Lisa

Twells, L.

Ufer, Christoph

Ulatowski, Lynn

Um, Sung Hee

Uribarri, Jaime

Ute, Alexy

Uto, Takuhiro

Utzschneider, Kristina M.

Vaisburg, Arkadii

Vajro, Pietro

Valdivielso, P.

Valent, Francesca

Valenti, Luca

Valerio, Costa

Vallgårda, Signild

van Baak, Marleen A.

van Ballegooijen, Hanne

van Heerden, P.V.

van Rossem, Lenie

van Schoor, Natasja M.

Vandenplas, Yvan

Vandewoude, Maurits F.J.

Vapaatalo, Heikki

Varela-Moreiras, G.

Vargas, Carmen Regla

Vecchia, Carlo

Vecoli, Cecilia

Vega, Renato S.A.

Velloso, Licio A.

Venn, Bernard

Verbeke, Kristin

Verduci, Elvira

Vermaak, Ilze

Vicente, Laura

Vickers, Mark

Vieira, A.

Vinciguerra, Manlio

Visavadiya, Nishant P.

Volterman, Kim

Von Hurst, Pamela von Lintig, Johannes

von Schacky, Clemens

von Wright, Atte

Wada, Jun

Wagner, Carol

Wagner, Erwin F.

Wagstaff, Carol

Wai, AU-YEUNG Kathy Ka

Walker, Alan W.

Walker, Celia G.

Wang, Chi Chiu

Wang, Gene-Jack

Wang, Hui

Wang, Jiawei

Wang, Lu

Wang, Peizhong Peter

Wang, Piwen

Wang, Ting

Wang, Xiaohui

Wang, Ying

Wang, Zhanxiang

Ward, Leigh

Ward, Mary

Wasse, Lucy

Waterhous, Therese S.

Watkins, Adam J.

Watkins, Richard R.

Wauquier, Fabien

Weaver, Connie

Weaver, Ian

Webb, Andrew J.

Webster, Jacqui

Weed, Harrison

Weinberg, Gene

Welsh, Jean

Westerterp, Klaas

Westerterp, Klaas R.

Whelan, Jay

Whisner, Corrie

Whisner, Corrie M.

Whiting, Susan

Widen, Elizabeth 


$\begin{array}{lll}\text { Wijnkoop, Lenoir } & \text { Xu, Fenglian } & \text { Zapolska-Downar, D. } \\ \text { Wilder-Smith, C.H. } & \text { Yakub, Mohsin } & \text { Zara, Vincenzo } \\ \text { Wilkinson, J.M. } & \text { Yamamoto, Hironori } & \text { Zarrelli, Armando } \\ \text { Williams, Anne } & \text { Yang, Chung S. } & \text { Zhang, Guirong } \\ \text { Williams, John } & \text { Yang, Daichang } & \text { Zhang, Xuehong } \\ \text { Williams, Nancy I. } & \text { Yang, Mhan-Pyo } & \text { Zhao, Liang } \\ \text { Williamson, Patricia } & \text { Yang, R.-H. } & \text { Zhao, Yuanxiang } \\ \text { Wilson, Gabriel } & \text { Ye, Sang-kyu } & \text { Zhao, Yuejen } \\ \text { Winter, Sebastian } & \text { Yeh, Shu-Lan } & \text { Zhou, Fang Qiang } \\ \text { Wlazlo, Nick } & \text { Yeung, Edwina } & \text { Zhou, Yifa } \\ \text { Włodarek, Dariusz } & \text { Ying, Danyang } & \text { Zhu, Meijun } \\ \text { Wong, Sze Choong } & \text { Yokoyama, Yoshihito } & \text { Ziouzenkova, Ouliana } \\ \text { Woo, Jessica Graus } & \text { Yu, Danxia } & \text { Zittermann, Armin } \\ \text { Worsley, Tony } & \text { Yun, Jong Won } & \text { Zlokovic, Berislav } \\ \text { Wu, Jian-Ping } & \text { Yung, Raymond } & \text { Zwart, Sara } \\ \text { Xian, Cory J. } & \text { Yurko-Mauro, Karin } & \\ \text { Xing, Mingzhao } & \text { Zamora-Ros, Raul } & \end{array}$

(C) 2015 by the authors; licensee MDPI, Basel, Switzerland. This article is an open access article distributed under the terms and conditions of the Creative Commons Attribution license (http://creativecommons.org/licenses/by/4.0/). 